\title{
Training Social Problem Solving Skills in Adolescents with High-Functioning Autism
}

\author{
Fatima A. Boujarwah, Hwajung Hong, \\ Rosa I. Arriaga, Gregory D. Abowd \\ School of Interactive Computing \\ Georgia Institute of Technology \\ Atlanta, GA USA \\ fatima, hhong31, arriaga, abowd@gatech.edu
}

\author{
Jackie Isbell \\ College of Education \\ Georgia State University \\ Atlanta, GA USAJackie Isbell \\ jisbell1@student.gsu.edu
}

\begin{abstract}
Adolescents and young adults with highfunctioning autism spectrum disorders (HFASD) have very different needs and abilities. Deficits in social skills and executive function, however, are generally considered defining characteristics of HFASD. Deficits in socialization often interfere with these individual's educational experience and quality of life, and explicit instruction is required to help them acquire ageappropriate social skills. We describe an approach to social skills training for adolescents and young adults with HFASD. Our design allows the user to role-play through social scenarios - such as going to the movie theatre - in a way that we believe may lead toward generalization. We also present the findings of an exploratory study in which 8 young adults with HFASD interacted with a prototype system. These findings showed that participants with HFASD respond favorably to the software, and that, in the future it will be important to integrate all aspects of a complete interventions into the software.
\end{abstract}

Keywords-component; Computer-assisted Instruction; Autism; Social Skills Training

\section{INTRODUCTION}

Adolescents and young adults with high-functioning autism spectrum disorders (HFASD) have very different needs and abilities. Deficits in social skills and executive function, however, are generally considered defining characteristics of HFASD. Deficits in socialization interfere with these individual's educational experience and quality of life, and explicit instruction is required to help them acquire ageappropriate social skills.

In this paper we explore the use of interactive software to provide social skills instruction. Specifically, we are interested in developing systems that can help adolescents and young adults with HFASD rehearse and learn social skills with reduced aid from parents, and other caregivers. Our objective is to develop technology that can be used to teach effective problem solving by allowing the individual to experience social situations and choose appropriate responses to unexpected events. The technology can also assist and support reflecting on those experiences, providing the individual with an opportunity to process information that can be recalled for later use.

Permission to make digital or hard copies of all or part of this work for personal or classroom use is granted without fee provided that copies are not made or distributed for profit or commercial advantage and that copies bear this notice and the full citation on the first page. To copy otherwise, to republish, to post on servers or to redistribute to lists, requires prior specific permission and/or a fee.

PERVASIVEHEALTH 2010, March 22-25, Munchen, Germany

Copyright $\odot 2010$ ICST 978-963-9799-89-9

DOI 10.4108/ICST.PERVASIVEHEALTH2010.8807
There is a paucity of research that includes adolescents and young adults with HFASD, but there are indications that they respond well to computer-assisted instruction. Indeed there is a general call for more technologies that specifically target social skills training[1]. We chose to target this population because they are underrepresented with respect to applicable therapies and are more likely to have complex social skill needs. For example, an adolescent with HFASD may want to go to a movie theatre without the assistance of a parent or guardian. Can a software system help that individual prepare for that social context, and furthermore help the individual learn a set of social skills that can be successfully generalized to the actual social setting? These are questions that we would like to answer with our research.

In this paper we present the first steps towards realizing an effective social skills training system. First, we describe an approach to social skills training for adolescents and young adults with HFASD. Our prototype system allows the user to role-play through social scenarios - such as going to the movie theatre - in a way that we believe may lead toward generalization. Second, we present the findings of an exploratory study in which eight young adults with HFASD interacted with our prototype system.

The paper is arranged as follows. First, we describe related work from the domains of special education, autism intervention, and computer-aided education. In Section 3, we describe our scenario-based social skills learning system, our design approach, and the various steps taken to customize the system for the target users. This is followed by a presentation of our study methodology. Finally, we present the findings of our exploratory study, and a discussion of their implications.

\section{BACKGROUND AND RELATED WORK}

\section{A. Background}

More than a half-century ago Kanner [2] and Asperger [3] first described individuals with autism spectrum disorders (ASD) as perplexing individuals. The Diagnostic and Statistical Manual, Fourth Edition, Text-Revised (DSM-IVTR) [4], used by practitioners and researchers in the United States, lists five subtypes of ASD under the category of Pervasive Developmental Disorders: Rhett's disorder, childhood disintegrative disorder, autistic disorder (autism), Asperger disorder/syndrome (AS), and pervasive 
developmental disorder, not otherwise specified (PDD-NOS). Characteristics commonly associated with all of the subtypes include deficits in the areas of communication and socialization, accompanied by restricted interests and repetitive behaviors. The complexity of the disorders included in the spectrum allows for individuals with ASD to be a heterogeneous group. It is possible for each person to present with a unique combination of characteristics with varying degrees of impairment. It is generally accepted that this population presents tremendous challenges for personnel responsible for planning educational services.

Some individuals present with mild characteristics and exhibit no intellectual disabilities. These high-functioning individuals with ASD are typically identified with HFASD, AS, or PDD-NOS. These individuals qualify for special education services when characteristics of their disorder interfere with learning in the general education setting.

Impaired social functioning is the central feature of all HFASD. A lack of social competency can result in significant difficulties in daily living, academic achievement, and poor adult outcomes related to employment and social relationships $[5,6]$. Researchers and educators have attempted to develop and implement interventions that lead to social competency. However, the results of one recent meta-analysis suggest that school-based interventions were minimally effective for children with ASD [7]. Some researchers conclude that, while many of the social skills interventions promoted skill acquisition, there was little evidence that the skills generalized to other settings $[7,8]$.

\section{B. Related Work}

Social skills training interventions are an important part of the education of children with Asperger's syndrome and HFASD. Due to the lack of a recognized best practice, educators use a variety of techniques, often in combination, to teach these skills. Power Card [9] is one example of a nontechnological intervention, but Social Stories ${ }^{\mathrm{TM}}[10]$ is the paradigm most commonly used. In Social Stories ${ }^{\mathrm{TM}}$ parents or teachers develop stories that are related to some event in the child's life. Each story is meant to allow the child to understand the situation so that they process information in vivo to maintain appropriate and adaptive behavior. These stories are written in a manner that is intended to be instructive with minimal demands for active child involvement [11].

One approach to social skill training that uses a combination of technological and non-technological practices is the Junior Detective Training Program [12], which consists of group social skills training, parent training, teacher hand-outs, and a computer game. The social competence that was sought was operationally defined as engaging in reciprocal positive interactions with others and responding appropriately to others' behavior. This program was tested with 44 students between the ages of 7 and 11. Parent-reported social skills of those in the treatment group improved from the clinically significant range to within normal range. These findings reinforce the importance of developing such software systems, and show that they are an effective way to teach social skills.

The "I can Problem-Solve" program [13] is a software system used with children between the ages of 5 and 9 . During the training session the trainer presented a problem situation and a solution via static and dynamic images. Children were then asked to suggest new solutions, and were reinforced with a variety of sensory or natural conditions (e.g. lines and spirals or a child jumping on a trampoline respectively). Children with ASD produced fewer solutions than neuro-typical children, but the number of solutions produced by children with ASD increased with repeated usage of the software.

In a meta-analysis of peer-reviewed social skill interventions varying from role-playing tasks, to technology interventions, Rao et al. [14] make several recommendations for maximizing the impact of social skill intervention. One recommendation is that social skill interventions should target children in the higher functioning range of the autism spectrum. Another important factor identified by Rao et al. is the content; they found that a key aspect lacking in the outcome of the studies reviewed was generalizability of the skills being learned beyond the training setting.

Other experimental technological approaches to autism intervention include virtual reality simulations, and virtual peers for language learning. Tartaro and Cassell [15] and Bosseler and Massaro [16] use virtual animated characters to invite language learning. Tartaro and Cassell in particular cite the advantages of using a virtual human over actual human interactors: virtual humans have the patience to interact with individuals with ASD. Parsons et al. [17] created a virtual reality environment to familiarize individuals with autism with social settings. This work involved learning to identify roles and procedures in a social environment. Our work differs by simulating the progression through a social situation in which the individual with ASD must engage in social skills. In addition, while other studies used single subjects, or samples of convenience, we collected data from a cohort of individuals in a school setting.

\section{DESIGN APPROACH}

Our system was built in Adobe Flash and Adobe Flex Builder, and is made up of three interactive scenarios; Going to a Movie, Going to a New Restaurant, and Unlocking the Door. The system is inspired by Social Stories ${ }^{\mathrm{TM}}$ [10], and is designed to present the user with real life social situation in which an unexpected obstacle arises. Social Stories ${ }^{\mathrm{TM}}$ presents the user with a relevant story, but is not interactive; individuals are simply required to passively view the story. We augment this approach in several ways. The scenarios are of differing levels of difficulty and familiarity to the users. The Unlocking the Door Scenario, for instance, presents a social situation that takes place at school. This scenario was considered to be the least complex due to the nature of the social problem the individual is faced with, and their familiarity with the setting. The Going to a New Restaurant Scenario presents a scenario that is set in the home environment and requires more planning than the first scenario. Lastly the Going to a Movie Scenario, is considered the most complex as it takes place in the community and involves both complex interpersonal interactions and a dynamic environment. These scenarios were chosen because of their relevance to the user, both in terms of content and setting, and because they allowed for varying levels of difficutly.

Each scenario has two main components, an experience section and a reflection section. In the experience section the 
user is guided as they navigate a situation and overcome an unexpected social obstacle. Then, in the reflection section, the user revisits the decisions they made during the experience portion, and is able to reflect upon how they successfully found a solution to the social problem they encountered. Throughout each scenario, an invisible recording system logs data related to the choices the user makes as they interact with the system and the time it takes them to make those choices. This data gathering feature allows us to understand how an individual progresses and differences between individuals. In the future, this component can also be used to individualize the experience of each user. Following is a description of the various key design criteria incorporated into the system.

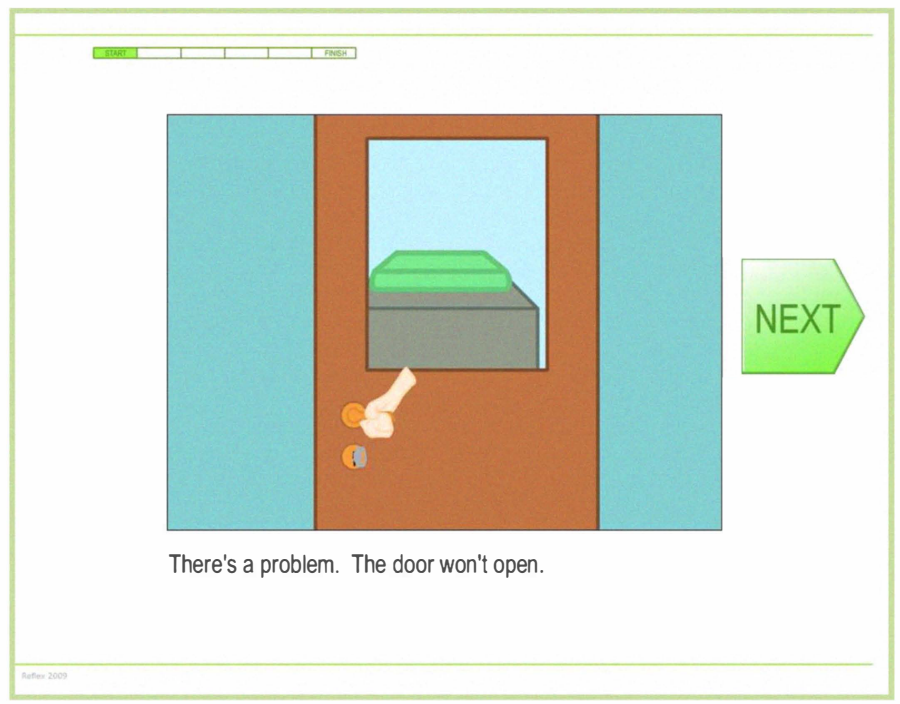

Figure 1. Step in the Unlocking the Door Scenario.

\section{A. The Experience Section}

In the experience section the system presents the social scenario through text, audio narration, and picture book style images that correspond to the specifics of the situation. (Fig. 1). This is similar to the approach used in Social Stories ${ }^{\mathrm{TM}}$ which uses cartoon figures and text-bubbles to represent speech. One advantage of this visual presentation is that it allows for the inclusion of the meaningful information, and the exclusion of distracting material. In addition, the system presents the scenarios in the first person perspective. This helps the user to identify with the character, and immerse themselves in the storyline. Individuals with autism prefer environments with high degrees of certainty, repetition, and predictability. Dealing with others in social settings introduces a high degree of uncertainty for those with autism. It is common for individuals with HFASD to rehearse for situations before hand. Unfortunately, rehearsal is not always effective as those with autism might learn cues specific to only one environment or one person [19]. Our visual design choices are meant to help avoid the learning of incorrect cues by limiting the information in each picture.

In order to ensure that the user acknowledges the narration, throughout the experience section all buttons are disabled until the audio narration has ended. In this way, the software prevents the user from clicking through the frames without perceiving the information that has been presented.

\section{1) Creating a Branching Story}

In order to make our system interactive, we approached each situation as a narrative with multiple paths, where a narrative is defined as a series of actions that describe how a situation unfolds. All the paths together resemble a branching story, which is a graph structure such that each node represents a segment of narrative and a choice point. The canonical branching story systems are Choose-Your-Own-Adventure novels. However, recent research has resulted in a spate of computational approaches to branching stories [18]. Our system can be considered to be an interactive narrative where each possible narrative is based on productive, unproductive, and counter-productive possible executions of social skills in specific contexts. The branching story we use in the experience portion consists of three major decision points in which the user has to make a series of choices to proceed to the next stage and ultimately successfully navigate the social situation.

At each branching point in the story the user is presented with a series of choices. The system plays all the choices individually then the user is taken to a screen where they are prompted to make a decision (Fig. 2). The user makes their choice by clicking on the image or button on the screen. This action takes them to a page where they can review the option before confirming their choice.

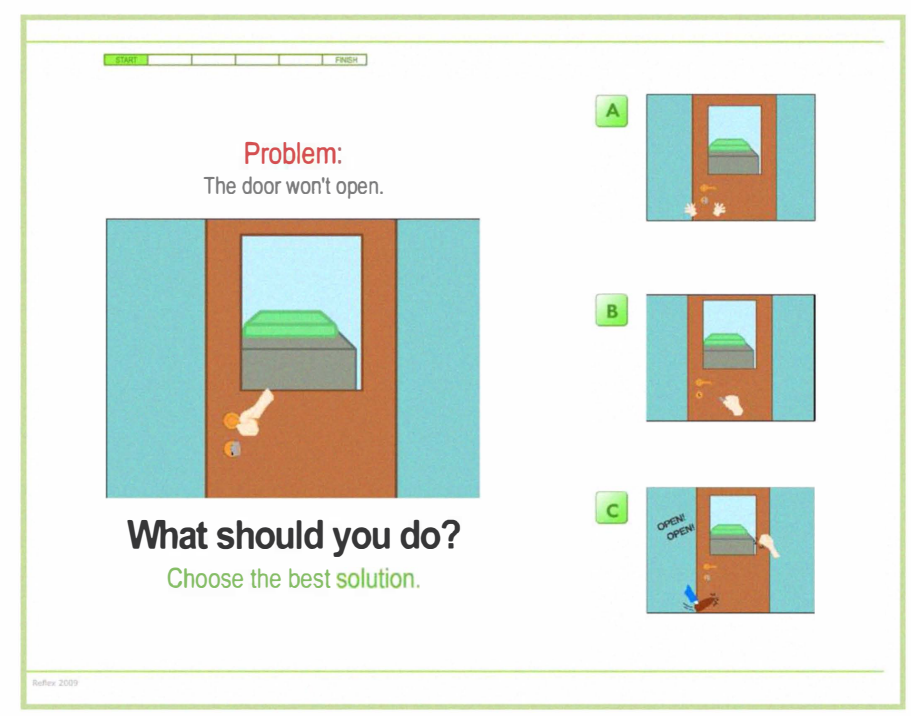

Figure 2. First Decision Point in the Unlocking the Door Scenario.

\section{2) Errorless Learning}

We follow an approach of errorless learning, which means that the user is not allowed to fail. When the user makes a choice that is considered unproductive or counter-productive the system explains the possible consequences of that action without using negative language, and directs the user to rethink their choice (Fig. 3). When the user returns to the decision point the undesirable choice that they have already explored is grayed out to prevent it from being chosen again. In this way the system provides immediate feedback and helps the user to correct their error. Errorless learning is often used with individuals with HFASD to avoid the possibility that they 
acquire incorrect skills; individuals with HFASD are extremely prone to repetition so it is essential to avoid reinforcing anything other than the desirable execution [19].

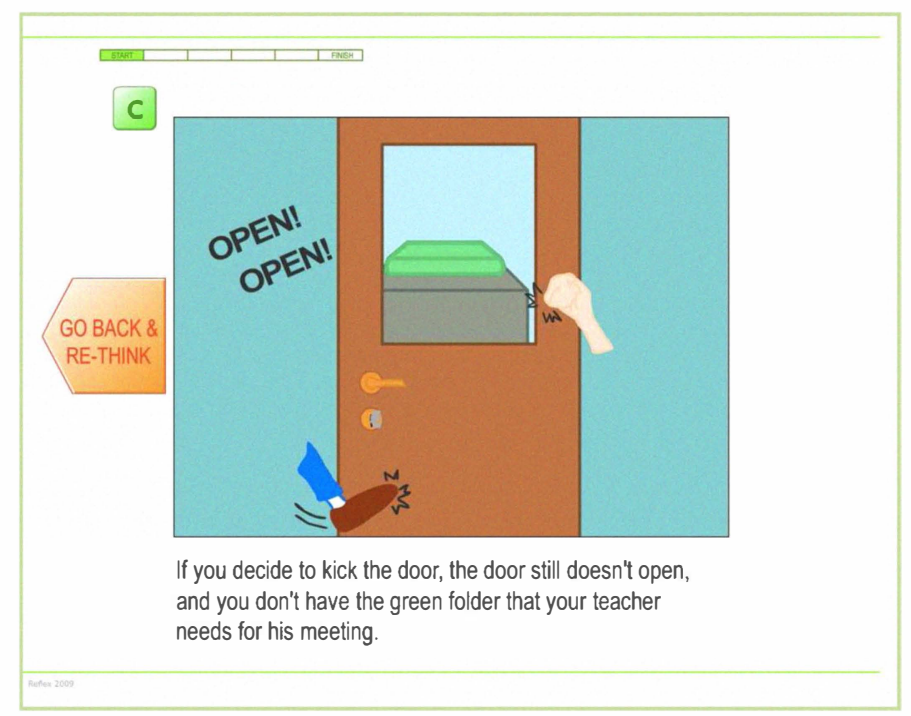

Figure 3. Explanation of the consequences of an undesirable choice.

\section{B. The Reflection Section}

Once the user has successfully navigated the experience portion of the scenario, they are asked to reflect on their decisions by recreating the social story. The user is presented with a puzzle piece for each decision they made, and is asked to recreate the story by sequencing the puzzle pieces correctly on a timeline (Fig. 4). The pictures used in this section are the same as those the user saw in the experience portion. For this portion the user is given both text and audio instructions.

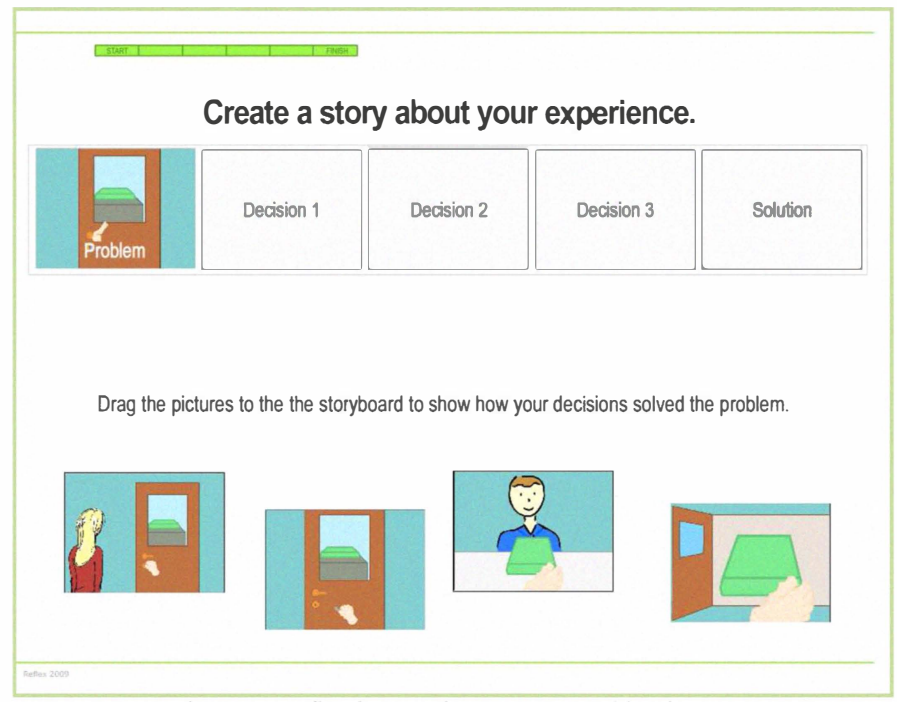

Figure 4. Reflection portion as presented by the system.

In the reflection portion the user is again given immediate feedback. If the user drags a puzzle piece to the wrong location on the timeline, they are prompted to "Try again" by a message that appears on the screen. When the user places a puzzle piece in the correct location the action is reinforced with a "Good job!" message. Once the user has successfully recreated the social story, the story is played back to them. Each picture appears on the screen with the corresponding text and audio narration. By prompting the user to revisit the story they created, the system reinforces the social problem solving skills that were used to solve the problem. In this way, the technology assists and supports the user as they reflect on the decisions they made and provides the user with an opportunity to process information that can be recalled for later use.

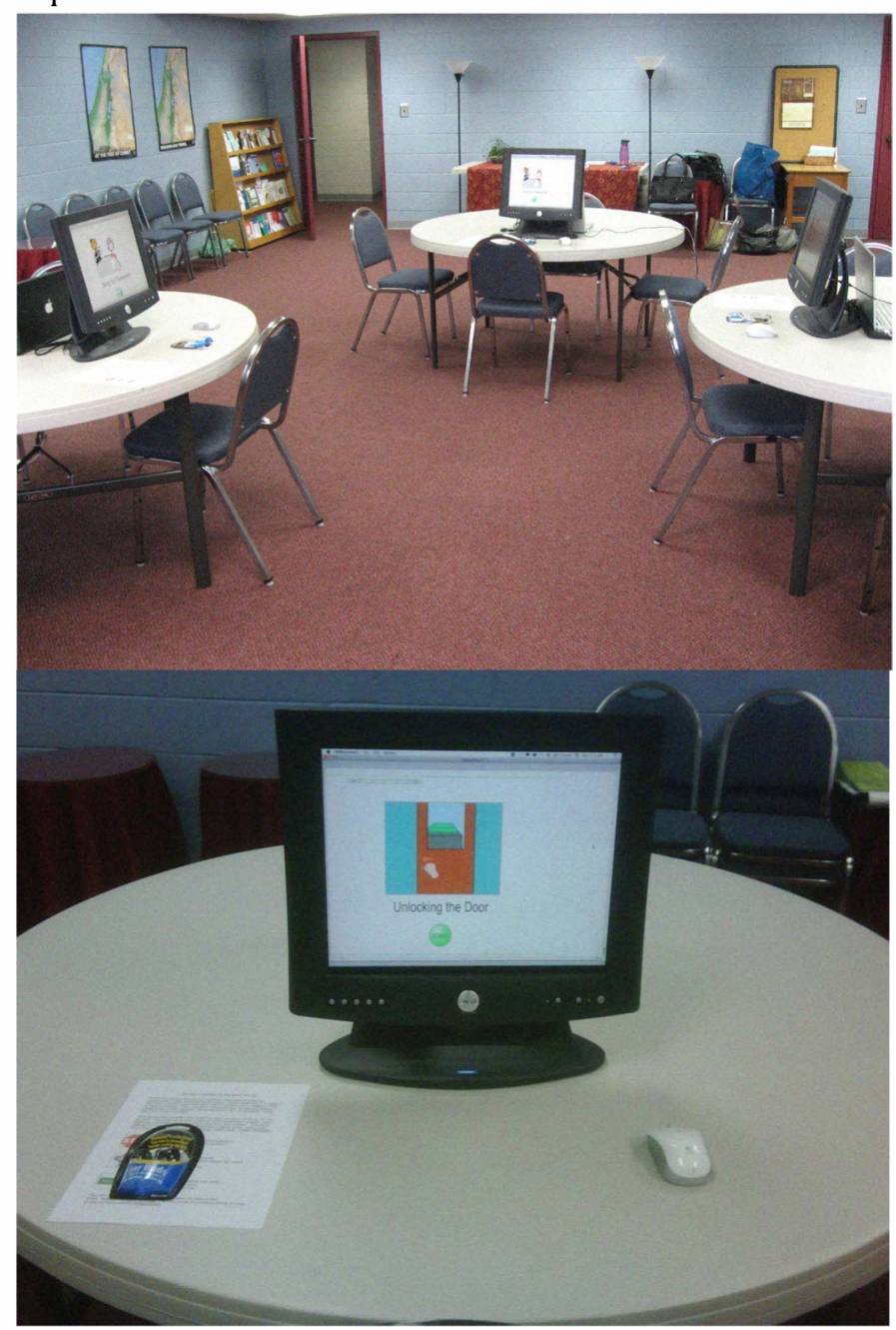

Figure 5. Setup of experimental equipment.

\section{Study Methodology}

In an effort to validate our design ideology, we conducted an exploratory study with eight individuals with HFASD. These individuals were students between the age of 13 and 19 years who were attending a special needs school in a major metropolitan city. Half of the participants were 18 years old or older, but were still in school due to their disability. We used the Test of Problem Solving for Children and Adolescents (TOPS2-A), which has an interview format with questions involving social situations. This test has been shown to correlate to actual problem-solving ability [20]. For this reason, it was used to assess the participants before they interacted with the system. None of the questions on TOPS2-A 
are directly related to the scenarios in our system. We also asked the participants to complete the Social Problem Solving Inventory (SPSI-R), which has a multiple-choice format, and is designed to determine an individual's perceptions of their own problem solving skills. We then asked the participants to complete each of the three scenarios in our prototype system. Our methodological procedure was as follows.

\section{A. Pre-tests}

Prior to beginning testing, all parents were given a description of the software and asked to give their consent to allow their child to participate in the study. Once parent consent had been obtained the participant's assent was collected. On the first visit, the researchers administered the standardized tests. All of the testing took place in the school in room usually used as a community room.

\section{1) TOPS- $2 \mathrm{~A}$}

The TOPS2-A test was administered individually to the participants. A researcher read a passage aloud while the participant read the same passage silently if they chose. The researcher then asked questions about the passage and instructed the participants to answer verbally. Two researchers were present during the testing and both scored the participant's responses. Immediately after each test was completed the two researchers discussed the scores to ensure inter-rater reliability and accuracy in the scoring. Since the TOPS- $2 A$ has only been validated for use with individuals age 12 to 17 and 11 months, the test was only administered to the four participants that were in this age range.

\section{2) SPSI}

The following week, before using the software, the participants were asked to complete the SPSI. The researcher read the questions aloud and asked the participant to respond verbally. Participants were allowed to complete the SPSI on their own if they wanted.

\section{B. Testing}

On the second week the hardware was set up in the same community room. Three computers were set up such that the participants had their backs to each other and would not distract each other (Fig. 5). This allowed the participants to complete the scenarios in parallel, thereby reducing the disturbance to their normal routine. The participants completed the scenarios in three groups, two groups of 3 , and a group of 2 . In the interest of hygiene, each participant was given a new headset that was labeled so that the participant could use them on all three days of the software testing. Since the software only required mouse interaction, participants were not provided with a keyboard (Fig. 5).

Each day before they began to interact with the software the researchers read the participants a short passage that introduced a problem solving process (Fig. 6). This passage explicitly presented the problem solving process that the participant is lead through in the software. Following the Social Story's, format this passage was presented on a sheet of paper with brightly colored icons. Each participant then completed one scenario per day for three consecutive days. We presented the scenarios in order of difficulty; asking the participants to complete the Unlocking the Door Scenario the first day, the Going to a New Restaurant Scenario the second day, and the Going to a Movie Scenario the third day. In order to reward the participants for their efforts, they were allowed to play a computer game after completing each day's scenario.

\section{Solving Problems in the Real World}

Sametimes things go wrong in the reaj world. When somethirg happens unexpectedly or when something doesn't happen the way pespie want it to happen, they hove $a$ problem. Sometimes when I can't complete a job or assignment that my teacher gives me, I have a probiem. Sometimes when my schedule changes unexpectedly, I hove a probiem. Sometimes when I car't do something I really want to do. I have a aroblem.

When people have a problem, they might feel angry, scared, or confused. If they get upset, they might rot be able to thirk of $c$ woy to solve their groblem. Some people use a strctegy to help then find a solution to their problem. A strategy helps people organize their thoughts and develop a pian of actian. Samie people follow these steps of a problem solving strategy called STOP. THINK, PROCEED:

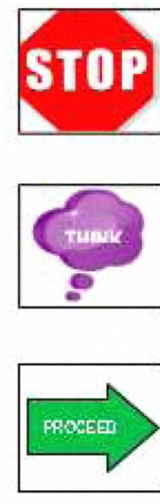

Take o few deep breaths and calm down
Ask yourself, "What is the probiem?" State the probler:

Think of some possible solutiors. Ask yourself. "Which solution will get the results I want?" Decide which solution to try.

The next rime I have o problem, I will try to follow the steps in the STOP. THINK, PROCEED strategy. I wili try to use this proslem solving strategy to help me find a solution to my problem.

Figure 6. Problem Solving Process as presented to the participants.

\section{RESULTS}

To enable a detailed discussion of the log data collected, only the results from the standardized testing, and the findings from the Unlocking the Door Scenario will be presented.

\section{A. Standardized Testing}

Only 2 of the 4 participants administered the TOPS- $2 A$ were able to successfully complete it, P3 and P6. P6 gave responses that resulted in a standard score in the low average to average range for his age of 15 years and 9 months. His test score resulted in an age equivalency of 12 years and 6 months. P3 gave responses that resulted in a standard score in the below average to low range for his age of 13 years and 5 months, and an age equivalency below the minimum measured by the test, which is 11 years and 2 months.

P4 attempted the test, but was unable to complete it. He became agitated and began repeating parts of the question as his response. For this reason, his testing was stopped and scoring his test was not possible. We were also unable to score P2's test. He had very limited language, and we were unable to complete his test because we could not be sure that he understood the passage or the questions. 
We only attempted to administer the SPSI to the 2 adolescent participants who successfully completed the TOPS2-A (P3 and P6). P6 was able to complete the SPSI independently, scoring 124 , which is in the above average range. P6 responses classified him as having a positive problem orientation, with an avoidant style of problem solving. P3, however, showed signs of stress part way through the survey and we did not ask him to complete it.

Only 2 of the participants over 18 were able to complete the SPSI. P8 was able to complete the inventory independently, scoring 89 , which is in the low average range. P7 was also able to complete the SPSI, however, he asked that a researcher read it to him, indicating that "reading is part of [his] disability." P7 scored 90 on the SPSI, which is in the average range. Both these participants were classified as having a negative problem orientation, and an avoidant style of problem solving. Of the 2 remaining participants over 18 , only P5 attempted the test, but began showing signs of distress almost immediately so we stopped the test. We did not feel that P1 had the verbal language ability to be able to complete the inventory, so we did not attempt it.

\section{B. Testing: Software Interaction, Scenario 1}

All the participants completed the scenario successfully during the first day of software testing. The logs collected by the software provided us with a detailed description of the participants' interaction with the system; recording button clicks and timings. As can be seen in Figure 7, 7 out of the 8 participants chose a path considered to be complex, in that the participant was required to navigate three decision points in order to successfully solve the social problem. In addition, all 7 of these participants chose the same complex path $(2 \mathrm{C}$, getting help from a teacher). There was a great deal of variation, however, in the time it took the participants to complete the scenario. Participant P5, for instance, completed the complex path in the shortest time, taking only 156 seconds, and P6 took the longest time, taking 252 seconds (Fig. 8).

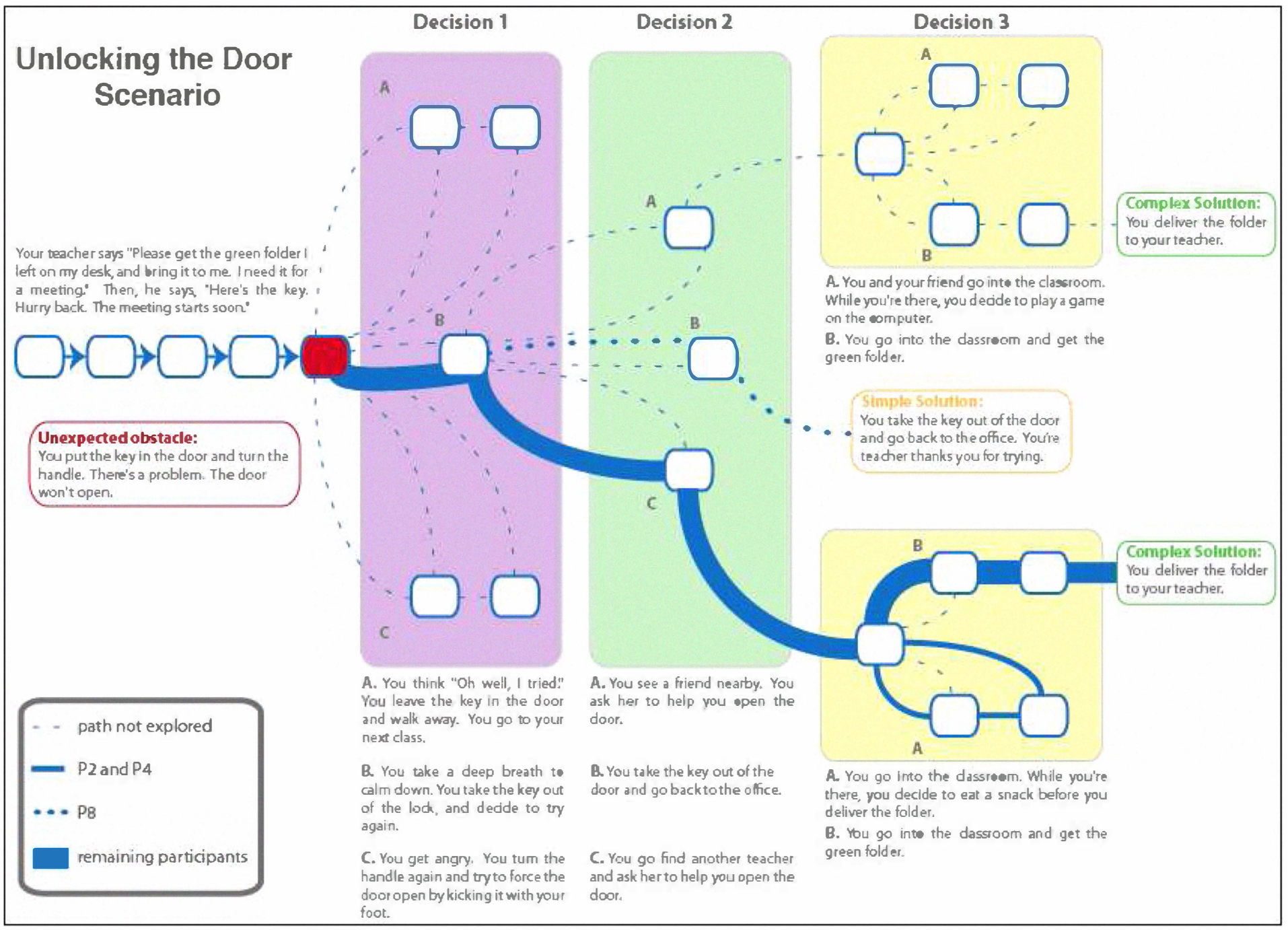

Figure 7. Paths chosen in scenario 1. 


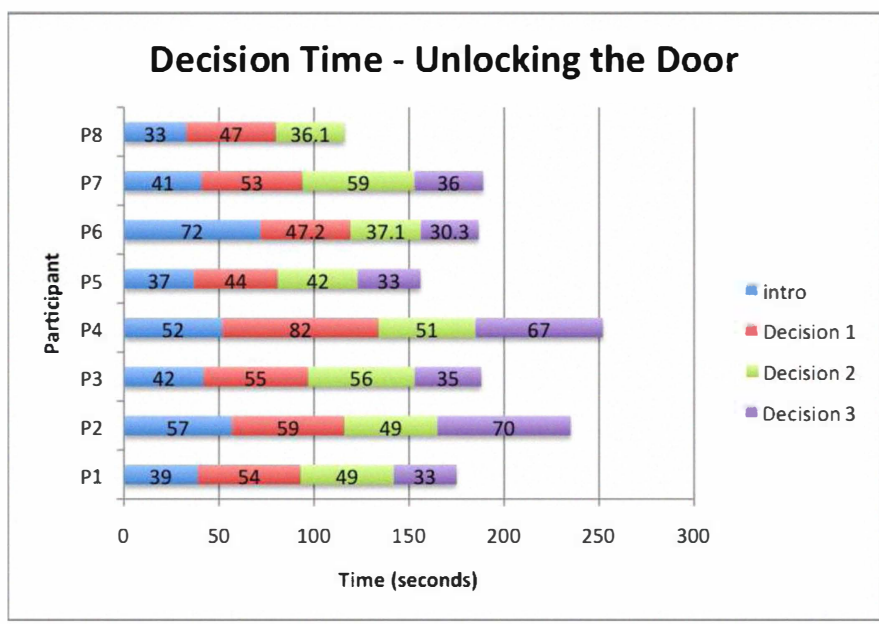

Figure 8. Time to complete each stage in scenario 1.

The logs also allowed for the analysis of data regarding the number of times a participant clicked a particular button. This enabled us to see which participants did not wait until the audio narration had completed before attempting to proceed. As can be seen in Table 1, this data also varied significantly across participants. In this case a low number indicates that the participant more often waited to hear the complete narration, and a zero in decision 3 indicates that the user chose the simple solution, and therefore did not have to complete the third stage of the scenario.

Log data was also collected from the Reflection portion of the software. This data showed longer than usual pauses in some of the participants' transitions from the Experience to the Reflection portion. Following the pause, however, very few errors were made. Six out of the eight participants (all except P2 and P7) made one or fewer errors before successfully populating the timeline.

\section{Post-Hoc Analyses:}

Following the conclusion of the study, the two researchers that were present during the standardized testing and throughout the software testing were asked to rank the participants with respect to their language abilities and visible characteristics of Autism. One of the researchers is a $\mathrm{PhD}$ student in Computer Science, and the other is a PhD student in Education with more than ten years of experience teaching children with special needs. Despite these differences in background, both the researchers ranked the participants in the exact same order. These rankings can be seen in Table 2, where 1 indicates the participants that was observed to have least noticeable characteristics of Autism and the most language and 4 indicates the participant perceived to have the most characteristics of Autism and the least language.

The participants were divided into those that were over 18 years of age and those that were minors. This allowed for the timing data and the click data to be correlated to the ranking. For the minors in the study, we found that in all three decisions the number of clicks was negatively correlated with the participants subjective ranking. We also found that the timing data was very strongly positively correlated with the ranking in the minor participants, with the total time having a 0.95

\begin{tabular}{lccccc} 
& Intro & Decision & Decision & Decision & Total \\
& & 1 & 2 & 3 & \\
\hline P1 & 5 & 3 & 3 & 4 & 15 \\
P2 & 5 & 3 & 3 & 7 & 18 \\
P3 & 5 & 3 & 3 & 5 & 16 \\
P4 & 5 & 5 & 4 & 7 & 21 \\
P5 & 5 & 6 & 7 & 6 & 24 \\
P6 & 5 & 12 & 15 & 19 & 51 \\
P7 & 5 & 4 & 3 & 3 & 15 \\
P8 & 8 & 6 & 7 & 0 & 21 \\
\hline
\end{tabular}

Table 1. Number of clicks logged in the Unlocking the Door Scenario.

\begin{tabular}{|cccc|}
\hline Minor & Rank & Over 18 & Rank \\
\hline P6 & 1 & P7 & 1 \\
P3 & 2 & P8 & 2 \\
P2 & 3 & P5 & 3 \\
P4 & 4 & P1 & 4 \\
\hline
\end{tabular}

correlation with the ranking. These correlations were not as clear in the data from the participants over 18, however.

\section{DISCUSSION}

One of the goals of this study was to have students learn the general problem solving process (Fig. 7) and then present the software in an effort to help them generalize this process. We found that the current presentation of the material in a paper format (like traditional Social Stories ${ }^{\mathrm{TM}}$ ) was not effective in getting the participants attention. We suspect that the participants were less interested in the format given when the computer was readily visible to them. In future studies we plan to incorporate this step into the computer software.

Only 3 of the 8 participants ( 1 minor, 2 over 18 ) were able to complete the standardized testing component of this study. The goal of using standardized testing measures was to have an objective measure of the students' problem solving ability before beginning our intervention.

Given that our sample size was small we cannot make claims about the general feasibility of using these two measures with a population with HFASD, however, it does indicate that we need to have an alternative manner of assessing problem solving skills. One consideration is that both of the current assessments required that the participants propose solutions, in other words they would need to recall information. Research indicates that for individuals with HFASD "recalling" information taxes their executive function and thus may be more difficult than other manners of responding [21]. Thus, in the future we plan to adapt the problem-solving test so that it is incorporated into the software, and participants are only required to recognize appropriate options (multiple-choice). This manner of response is less taxing cognitively and may result in a higher rate of successful completion. This will enable us to have a better means of assessing social skills acquisition. 
Despite the difficulties we encountered with the standardized testing, our experience with individuals with HFASD made us confident that our participants would interact more favorably with the technology. Our expectations were confirmed during the study, as all the participants were able to successfully complete all three scenarios. This supports the idea that the software was able to provide the scaffolding necessary to enable even the participants that struggled with the testing to be successful during the software intervention.

In the Unlocking the Door Scenario, participants had the choice of either giving up and returning the key, or asking a friend or teacher for help. The latter is more complex because it requires interpersonal interaction, and the navigation of a third decision point. Seven out of eight participants chose the same complex solution, which involved the user asking a teacher to help them open the door (Fig. 7). Our study was conducted at a school in which the students interact one-on-one with a counselor for most of the day. For this reason, we believe that this choice is a reflection of the environment in which these participants find themselves.

The results indicated that longer pauses were logged at the transition between the experience and the reflection sections. We believe that this is the result of the fact that the actions expected from the user in this section were notably different from those in the experience portion. All the participants were able to complete the reflection portion successfully indicating that some thought was all that was required.

The subjective ranking allowed us to analyze the log data further (Table 2). We found that the minors that were ranked higher were more impatient and did not wait to hear the audio. We believe that this is likely because these participants were able to read the text quickly, and therefore were ready to proceed before the audio had completed.

The correlations between subjective participant ranking and log data for the participants over 18 were not as telling. This can be explained in several ways. In conversation with P1 and his counselor, for instance, we learned that, despite exhibiting limited verbal language, P1 was a proficient reader and Scrabble ${ }^{\mathrm{TM}}$ player. These skills enabled him to benefit from the text provided in the software and navigate the scenarios successfully. Similarly P7 exhibited particular interests in technology and suggested that we modify the system to allow for those that can, to proceed faster. Knowing that the buttons would not respond until the sound had completed, he chose to wait before attempting to proceed.

\section{CONCLUSION}

The population of young adults with high functioning autism spectrum disorder (HFASD) is growing. Many of these individuals can function effectively and autonomously, but need assistance to handle the complexities of society. This study suggests that participants with HFASD responded favorably to a prototype software system designed to aid in training social problem solving skills. This is in line with many other studies that have found that individuals with HFASD do well with computer-assisted interventions [1, 12, $13,14,15]$. It also suggests that students may do better with interventions that are wholly computer based. We found that participants struggled with standardized social skills tests, and were not interested in attending to information that was presented in a paper format, but were eager to get to work on the computer. As expected the log data provided us with insights into the process that the individuals used to solve a given scenario, suggesting that software versions of "social story" like interventions are well suited to analyses. This study also shows that a computer based intervention can be adapted to be used in a school setting and that students could, in fact, have a period where they are able to engage in an intervention then go back to their normal routine.

\section{FUTURE WORK}

It has been our goal to develop a software system that enables individuals with HFASD to experience and reflect on social problem. Now that the feasibility of the design ideology has been validated, our next step will be to develop a full-scale system that encompasses all aspects of an intervention, including software based assessment, and many diverse scenarios. This will allow us to assess the impact that the software has on the development of the participants' social problem solving skills.

\section{ACKNOWLEDGMENT}

We would like to thank Mark Riedl for his input on the creation of branching stories. We would also like to thank Waleed Manzoul for lending us his voice for the narration of the scenarios.

\section{REFERENCES}

[1] C. Putman. and L. Chong. Software and technologies designed for people with autism: What do users want? In Proc. of ASSETS 2008.

[2] L. Kanner, L. Autistic disturbances of affective content. The Nervous Child, 2: 217-250. 1943.

[3] H. Asperger. Die "autistichen psychopathen" in kindasalter. Archiv fur Psychiatrie and Nnervenkrankheiten, 117: 76-136. 1944.

H. Asperger. The Autistic Psychopathy of Childhood. Archive for Psychiatry and Nervous Diseases, 2:217-250. 1943.

[4] American Psychiatric Association. Diagnostic and Statistical Manual of Mental Disorders (4th ed., text revised). Washington D.C, 2000.

[5] A. Klin, and F.R. Volkmar. Asperger syndrome and external validity. Child and Adolescent Clinics of North America, 12(1): 1-13. 2003.

[6] P. Howlin. Outcome in high-functioning adults with autism with and without language delays: Implications for the differentiation between autism and Asperger syndrome. Journal of Autism and Developmental Disorders, 33: 3-13. 2003.

[7] S. Bellini, J.K. Peters, L. Benner, and A. Hopf. A meta-analysis of school-based social skills interventions for children with autism spectrum disorders. Remedial \& Special Education, 28: 153-162. 2007.

[8] L.M. Elder, L.C. Caterino, J. Chao, D. Shacknai, and G. DeSimone. The efficacy of social skills treatment for children with asperger syndrome. Education and Treatment of Children, 29: 635-663. 2006.

[9] E. Gagnon. The Power Card: Using Special Interests to motivate Children and Youth with Asperger Syndrome and Autism. Shawnee Mission, KS: Autism Asperger Publishing Company.

[10] C. Gray. Social Stories Unlimited: Social Stories and Comic Strip Conversations. Jenison, MI: Jenison Public Schools, 1995.

[11] G. Reynhout, \& M. Carter. The use of Social Stories by teachers and their perceived efficacy. Research in Autism Spectrum Disorders, 3, 232-251. 2009

[12] R. Beaumont and K. Sofronoff. A multi-component social skills intervention for children with Asperger's Syndrome: The Junior Detective Training Program. The Journal of Child Psychology and Psychiatry, 49(7): 743-753. 2008. 
[13] V. Bernard-Optiz, N. Sriram, and S. Nakhoda-Sapuan. Enhancing social problem solving in children with autism and normal children through computer-assisted instruction. Journal of Autism and Developmental Disorders, 31(4): 377-384. 2001.

[14] P.A. Rao, D.C. Beidel, and M.J. Murray. Social skills interventions for children with Asperger's syndrome or high-functioning autism: A review and recommendations. Journal of Autism and Developmental Disorders, 38(2): 353-361. 2008.

[15] A. Tartaro \& Justine Cassell. Playing with virtual peers: bootstrapping contingent discourse in children with autism. In Proc. of the Int. Conf. of the Learning Sciences (ICLS). 2008.

[16] A. Bosseler \& D. Massaro. Development and evaluation of a computeranimated tutor for vocabulary and language learning in children with autism. Journal of Autism and Developmental Disorders, 33(6): 653672. 2003.
[17] S. Parsons, P. Mitchell, \& A. Leonard. The use and understanding of virtual environments by adolescents with autistic spectrum disorders. Journal of Autism and Developmental Disorders, 34(4): 449-466. 2004.

[18] M. Riedl, Andrew Stern, Don Dini, and Jason Alderman. Dynamic experience management in virtual worlds for entertainment, education, and training. International Transactions on Systems Science and Applications, 4(2), 2008

[19] L. J. Heflin \& P. A. Alberto. Establishing a Behavioral Context for Learning with Students with Autism. Focus on Autism and Other Developmental Disabilities, 16, 93-101.

[20] T.J. D'Zurilla, A.M. Nezu, A. Meydeu-Olivares. Social Problem Solving Inventory-Revised. The Cognitive Center Foundation. 2002.

[21] D.F. Griswold, G.P. Barnhill, B.S. Myles, T. Hagiwara, and R.L. Simpson. Asperger syndrome and academic achievement. Focus on Autism and other Developmental Disabilities, 17: 94-102. 2 